\title{
Quantitative detection of DNMT3A R882H mutation in acute myeloid leukemia
}

Rimma Berenstein, Igor Wolfgang Blau, Nikola Suckert, Claudia Baldus, Antonio Pezzutto, Bernd Dörken and Olga Blau*

\begin{abstract}
Background: DNMT3A mutations represent one of the most frequent gene alterations detectable in acute myeloid leukemia (AML) with normal karyotype. Although various recurrent somatic mutations of DNMT3A have been described, the most common mutation is located at R882 in the methyltransferase domain of the gene. Because of their prognostic significance and high stability during disease evolution, DNMT3A mutations might represent highly informative biomarkers for prognosis and outcome of disease.
\end{abstract}

Methods: We describe an allele-specific PCR with a Blocking reagent for the quantitative detection of DNMT3A R882H mutation providing the possibility to analyze the quantitative amount of mutation during the course of disease. Next, we analyzed 62 follow-up samples from 6 AML patients after therapy and allogeneic stem cell transplantation (alloSCT).

Results: We developed an ASB-PCR assay for quantitative analysis of R882H DNMT3A mutation. After optimization of blocker concentration, a R882H-positive plasmid was constructed to enhance the accuracy of the sensitivity of quantitative detection. The assay displayed a high efficiency and sensitivity up to $10^{-3}$. The reproducibility of assay analyzed using follow-up samples showed the standard deviation less than $3.1 \%$. This assay displayed a complete concordance with sequencing and endonuclease restriction analysis. We have found persistence of DNMT3A R882 $\mathrm{H}$ mutations in complete remission (CR) after standard cytoreduction therapy that could be indicating presence of DNMT3A mutation in early pre-leukemic stem cells that resist chemotherapy. The loss of correlation between NPM1 and DNMT3A in CR could be associated with evolution of pre-leukemic and leukemic clones. In patients with CR with complete donor chimerism after alloSCT, we have found no DNMT3A R882H. In relapsed patients, all samples showed an increasing of both NPM1 and DNMT3A mutated alleles. This suggests at least in part the presence of NPM1 and DNMT3A mutations in the same cell clone.

Conclusion: We developed a rapid and reliable method for quantitative detection of DNMT3A R882H mutations in AML patients. Quantitative detection of DNMT3A R882H mutations at different time points of AML disease enables screening of follow-up samples. This could provide additional information about the role of DNMT3A mutations in development and progression of AML.

Keywords: AML, DNMT3A mutations, Quantitative PCR

\footnotetext{
* Correspondence: olga.blau@charite.de

Department of Hematology, Oncology and Tumorimmunology, Charité

University School of Medicine, Hindenburgdamm 30, 12200 Berlin, Germany
} 


\section{Introduction}

Somatic mutations in the DNA nucleotide methyltransferase 3A gene (DNMT3A) have been reported approximately in $22 \%$ of de novo acute myeloid leukemia (AML) and $36 \%$ of cytogenetically normal AML [1]. Mutations in DNMT3A were first described by Ley et al. using whole genome sequencing [2]. DNMT3A belongs to the mammalian methyltransferase gene family which is responsible for tissue-specific gene expression [3]. DNA methyltransferases are the key enzymes for genome methylation, which plays an important role in epigenetically regulated gene expression and repression. DNMT3A together with other methyltransferases conducts de novo methylation of cytosine residues in $\mathrm{CpG}$ islands by the enzymatic addition of methyl residues from S-adenosyl-L-methionine to the 5-carbon atom of the cytosine ring. $\mathrm{CpG}$ islands are often located proximate to gene promoters thereby regulating their activation. Actively transcribed genes display unmethylated CpG islands which supports the euchromatin structure whereas methylated CpG islands are associated with untranscribed genes stabilizing the heterochromatin structure $[4,5]$. Cancer genomes are most commonly characterized by global DNA hypomethylation. However, cancer cells also typically exhibit distinct regions of DNA hypermethylation, which are particularly well characterized in the CpG islands of promoter regions of tumor-suppressor genes. Although various recurrent DNMT3A mutations have been described, the most common mutation affects residue R882 within the methyltransferase domain. DNMT3A mutations are typically heterozygous $[2,6]$.

The biology of DNMT3A is not fully understood. HolzSchietinger et al. reported that mutations in DNMT3A could retard its function by multiple mechanisms as changes in the catalytic properties, its processivity and the disruption of interaction with binding partners [7]. Furthermore, Russler-Germain et al. found that mutations in the position R882 inhibit the formation of active tetramers of DNMT3A [8]. The impaired function of mutated DNMT3A leads to a hypomethylated genome of myeloid cells possibly promoting leukemogenesis and influencing disease outcome [9].

Since the DNMT3A mutations are present in the early pre-leukemic cells, this alteration seems to be a "founder" mutation, which can be implicated as functional components of AML evolution [10,11]. DNMT3A mutations are highly associated with mutations in the nucleophosmin 1 gene (NPM1), fms-related tyrosine kinase 3 gene (FLT3), and isocitrate dehydrogenase 1 gene $(I D H 1)[12,13]$.

Several studies reported a negative prognostic impact of DNMT3A mutations [12-16]. Prognostic effect is known to depend on certain biological factors as well as a combination of cytogenetics and other mutations such as those in FLT3 and NPM1.
Some authors have found stability of DNMT3A mutations during the course of disease; therefore those aberrations could be potential marker for minimal residual disease (MRD). Furthermore, the presence of DNMT3A mutations seems to be associated with the incidence of FLT3-ITD-positive clones at relapse possibly influencing the responsiveness of FLT3-positive cases to chemotherapy $[17,18]$. Last published data have demonstrated that DNMT3A mutations are also detectable in AML patients in long-term complete remission (CR) and can occur in pre-leukemic stem cells $[10,11,19]$. The identification of pre-leukemic cells with genetic mutations in CR has important implications for the MRD monitoring. Moreover, the persistence of DNMT3A mutations in CR may have important implications for the management of AML.

Recent discoveries utilizing whole-exome sequencing in a large cohort of persons, unselected for cancer or hematologic phenotypes have demonstrated somatic mutations in significant proportion of persons particularly which older than 65 years. Moreover, DNMT3A gene together with TET2, ASXL1, and PPM1D had disproportionately high numbers of somatic mutations $[20$, 21]. The data suggest that mutations in pre-leukemic cells could precede leukemia. Furthermore, DNMT3A mutations could drive clonal expansions. Based on these data, DNMT3A mutation might represent highly informative biomarkers for AML. Thus, a negative prognostic impact and, in addition, conflicting reports on the potential role of DNMT3A mutations for the evolution of leukemic stem cell, require fast, reliable, quantitative and available methods for detection of mutation.

Sanger sequencing is well-established but not very sensitive as well as time-consuming and cost-intensive method. HRM analysis provides the possibility of high throughput screening of mutations, but data interpretation occasionally can be difficult. Therefore, good validated controls and standards are needed. Previously we reported a rapid and reliable restriction fragment length polymorphism based method for the qualitative detection of DNMT3A $\mathrm{R} 882 \mathrm{H}$ mutation [22]. Current quantitative established assays have low sensitivity and therefore cannot be used as reliable methods for MRD diagnostic. Here, we describe an Allele-Specific PCR with a Blocking reagent (ASBPCR) for the quantitative detection of DNMT3A R882H mutation with sensitivity up to $10^{-3}$ providing the possibility to analyze the quantitative amount of this mutation for routine diagnostic during the course of disease.

\section{Methods}

\section{Patient and control materials}

Bone marrow (BM) samples from 16 newly diagnosed AML patients, 8 DNMT3A positive and 8 DNMT3A wild type (wt), were included in the study. In addition, we analyzed 62 follow-up samples from 6 DNMT3A positive 
patients after chemotherapy and allogeneic stem cell transplantation (alloSCT). All patients were treated at the University Clinic Charité from September 2009 to May 2013. Diagnoses were established according to the WHO criteria [23]. Written informed consent was obtained from all patients in accordance with the Declaration of Helsinki and the ethical guidelines of the Charite University School of Medicine, which approved this study. In 6 AML patients included in the follow up study, induction therapy consisted of " $7+3$ " therapy with Cytosine Arabinoside and Daunorubicine. Second part of induction with " $7+3$ " started at day 22 , if at day 15 count of BM blasts were reduced. Once complete remission (CR) was achieved, 2-4 courses of consolidation chemotherapy with high-dose Cytosine Arabinoside were administered. At 1-st CR or at 2-nd CR patients were allocated to alloSCT.

Control material for DNMT3A mutation included DNA from K562 cell line (wt) and constructed plasmid which contains the R882H mutation.

\section{DNA extraction}

Mononuclear cells (MNCs) from BM aspirates were isolated using Ficoll density centrifugation as described [24]. DNA was extracted using Allprep DNA/RNA mini kit (Qiagen) from $1 * 10^{7} \mathrm{MNCs}$ as recommended by the manufacturer. DNA yields ranged from 50 to $300 \mathrm{ng} / \mu \mathrm{l}$.

\section{Allele-Specific PCR with a Blocking reagent (ASB-PCR)}

The ASB-PCR assay was designed using Primer3, Oligocalc and UCSC software. We used an allele-specific reverse primer containing the mutational spot $(\mathrm{R} 882 \mathrm{H} \mathrm{G}>\mathrm{A})$ at its 3 '-end. The blocking sequence was developed complementary to the wt allele. The discriminating base was located in the middle of the blocker. To prevent elongation by Taq polymerase, a phosphate group was added to the 3 '-end of the blocker. For fluorescence detection, a TaqMan probe was used (Fig. 1). All primer sequences are listed in Table 1. Further properties, such as melting temperature were developed in accordance with Morlan et al. [25]. The reaction mixture contained $12.5 \mu \mathrm{l} 2 \times \mathrm{Ab}-$ solute qPCR Mix (Applied Biosystems), 10 pmol of each forward and reverse allele-specific primer, $40 \mathrm{pmol}$ of ASBBlocker, 5 pmol of probe, and $30 \mathrm{ng}$ of DNA in a final reaction volume of $25 \mu \mathrm{l}$. Reaction was run at $95{ }^{\circ} \mathrm{C}$ for $10 \mathrm{~min}$ followed by 40 cycles of denaturation at $95^{\circ} \mathrm{C}$ for $20 \mathrm{~s}$ and annealing/elongation at $67^{\circ} \mathrm{C}$ for $45 \mathrm{~s}$ on a Rotor Gene 6000 Real-Time PCR Cycler (Qiagen).

\section{Plasmid preparation}

For absolute quantification, a plasmid containing the DNMT3A R882H G > A mutations was constructed. The template was amplified using ASB-PCR primers, and the fragment size ( $94 \mathrm{bp}$ ) was analyzed on a $1.5 \%$ agarose gel. The plasmid was prepared using the TOPO TA cloning Kit (Invitrogen) and chemically competent E.coli as per the manufacturer's instructions. Fragment insertion was checked using DNA sequencing with provided M13 forward and reverse primers.

\section{DNA sequencing and endonuclease restriction analysis of DNMT3A mutations}

PCR sequencing reaction was performed as previously described [22]. Amplified products were purified using the PCR Purification Kit (Qiagen) according to the manufactures instruction. Sequencing was performed using ABI310 Genetic Analyzer (Applied Biosystems), and data were analyzed using DNA Sequencing Analysis Software v.5.2.0. Endonuclease restriction analysis of DNMT3A $\mathrm{R} 882 \mathrm{H}$ mutation was performed using Fnu4HI (New England Biolabs) as previously reported [22].

\section{Qualitative and quantitative evaluation of mutations}

Qualitative evaluation for presence of NPM1, DNMT3A, $I D H 1$ and $I D H 2$ mutations were performed by Sanger sequencing using ABI310 Genetic Analyzer (Applied Biosystems) as previously described [22]. FLT3-ITD and FLT3 D835Y were quantified by fluorescence fragment analysis on a 310 Genetic Analyzer (Applied Biosystems) as previously described [26]. Quantitative analysis of presence NPM1 mutations was performed by real-time PCR using Rotor Gene 6000 Real-Time PCR Cycler (Qiagen). The reaction mixture contained $12.5 \mu \mathrm{l} 2 \times$ Absolute qPCR Mix (Applied Biosystems), 30 pmol of each forward and reverse allele-specific primer, $10 \mathrm{pmol}$ of forward and 6 pmol of reverse HCK probes, 25 pmol of each FAMMGB and HEX probes, and 50 ng of DNA in a reaction

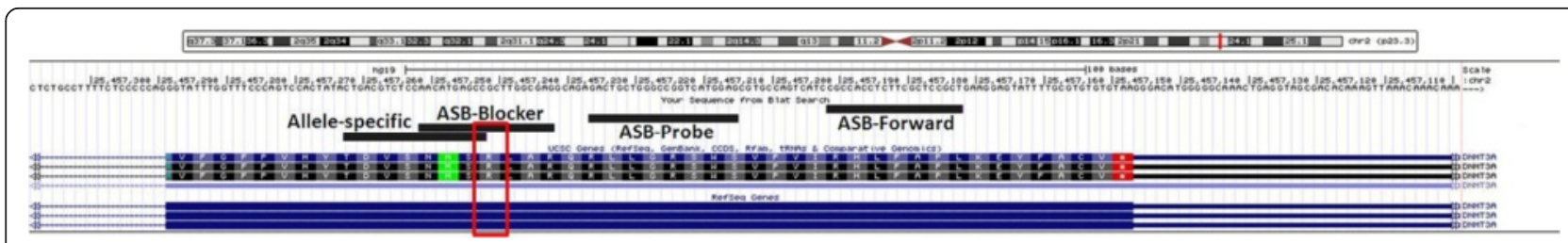

Fig. 1 Assay design of ASB-PCR. Primers and probe are located in exon 23 of DNMT3A. The allele-specific primer contains the mutational spot at its $3^{\prime}$-end whereas the wt spot is incorporated in the middle of the blocker (red box). Fluorescence detection was performed with a TaqMan probe which was designed near to the forward primer (13 bp distance) 
Table 1 Oligonucleotides used in this study

\begin{tabular}{llll}
\hline Name & Sequence & Application & Tm [ ${ }^{\circ}$ ] \\
\hline DNTM3A-Ex23F & 5'-GTGTGGTTAGACGGCTTCC & Sequencing & 59.5 \\
DNMT3A-Ex23R & 5'-CTCTCCCACCTTCCTCTG & Sequencing & 59.5 \\
ASB-F & 5'-CAGCGGAGCGAAGAGGTG & ASB-PCR & 60.8 \\
Allele-specific & 5'-CGTCTCCAACATGAGCCA & ASB-PCR & 56.3 \\
ASB-Blocker & 5'-CATGAGCCGCTTGGCGAG-PH & ASB-PCR & 60.8 \\
ASB-Probe & 5'-FAM-CTCCATGACCGGCCCAGCAGTC-BBQ & ASB-PCR & 69.5 \\
\hline
\end{tabular}

The mutational spot in the allele-specific primer and blocker sequence is bold and punctuated. ASB, indicates Allele-Specific PCR with a Blocking reagent; DNMT3A, indicates DNA methyltransferase 3 gene

final volume of $25 \mu$ l. Reaction was run at $95{ }^{\circ} \mathrm{C}$ for 15 min followed by 45 cycles of denaturation at $95^{\circ} \mathrm{C}$ for $15 \mathrm{~s}$ and annealing/elongation at $60^{\circ} \mathrm{C}$ for $1 \mathrm{~min}$.

\section{Results}

ASB-PCR assay performance

At first, we have tested the performance of ASB-PCR by standard PCR amplification and agarose gel electrophoresis.
Specificity of ASB-PCR was verified using Sanger sequencing. To determine the optimal blocker concentration, PCR was performed with $40 \mu \mathrm{M}$ and $80 \mu \mathrm{M}$ of blocker, successively. A specific amplification of mutated DNA was determined producing a PCR fragment of approximately $94 \mathrm{bp}$. DNA of the wt sample was also amplified, but with a considerably lower efficiency compared to R882H-positive DNA (Fig. 2.ai). No significant

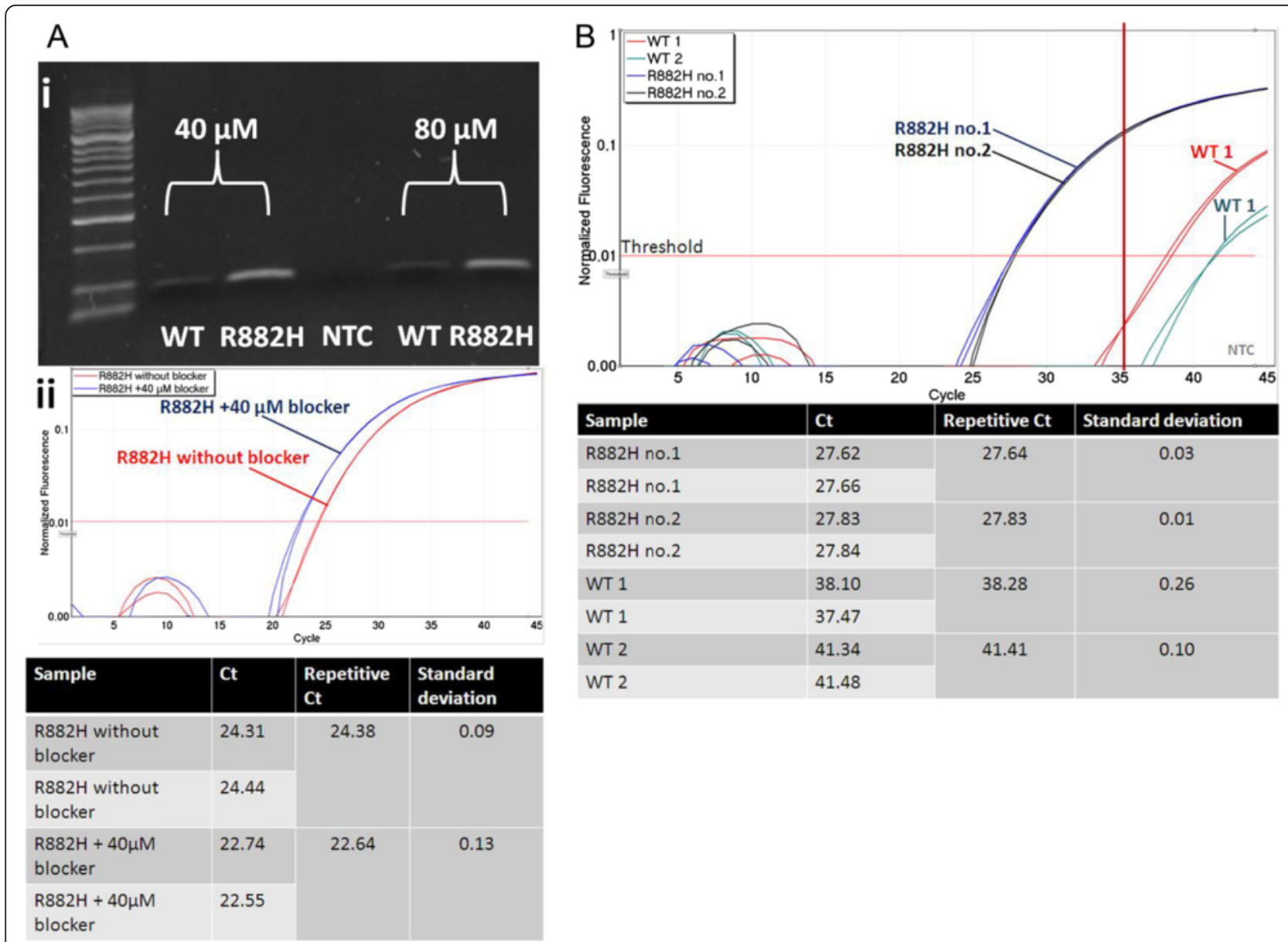

Fig. 2 Performance of ASB-PCR. ai Qualitative analysis of the specificity of ASB-PCR. Enhancement of blocker concentration to $80 \mu \mathrm{M}$ showed no significant change in the amplification properties. aii Quantitative analysis of Ct-changes induced by addition of $40 \mu \mathrm{M}$ blocker. $\mathbf{b}$ Quantitative analysis displayed a $C_{t}$ difference of 11-14 cycles between R882H mutated DNA and wt DNA. NTC, non-template control; wt, wild type 
difference was seen for PCR amplification with $40 \mu \mathrm{M}$ and $80 \mu \mathrm{M}$ of blocker. However, the presence of $40 \mu \mathrm{M}$ blocker in the reaction led to detection of $\mathrm{R} 882 \mathrm{H}$ around $2 \mathrm{Ct}$ 's earlier compared to the assay without blocker (22.64 vs. 24.38; Fig. 2.aii). Therefore, $40 \mu \mathrm{M}$ of blocker were used for further applications. Next, we determined the quantitative performance of the assay (Fig. 2.b). The fluorescence signal of mutated samples exceeded the threshold 11-14 cycles earlier as compared with wt samples $(27.64 / 27.83$ vs. 38.28/41.41). Based on this analysis, we chose a $\mathrm{C}_{\mathrm{t}}$-value of 35 as the cut-off limit.

Subsequently, the sensitivity of ASB-PCR was studied. First, a dilution series of genomic DNA was prepared ranging from $50 \mathrm{ng}$ to $1 \mathrm{ng}$ (Fig. 3.a). Differentiation between wt and $\mathrm{R} 882 \mathrm{H}$ samples was possible for all dilution steps. One ng of R882H DNA was detected 4 cycles earlier compared with $30 \mathrm{ng}$ of wt DNA (32.09 vs. 36.31). In addition, a dilution series of R882H-positive DNA with wt DNA was analyzed (Fig. 3.b). Discrimination of $1 \%$ of $\mathrm{R} 882 \mathrm{H}$-positive DNA was possible exceeding the threshold approximately 3 cycles earlier as the wt DNA (33.60 vs. 36.31). The cut-off limit of $\mathrm{C}_{\mathrm{t}}=$ 35 was constant for these analyses. To enhance the accuracy of the sensitivity determination of quantitative detection of the mutation, a $\mathrm{R} 882 \mathrm{H}$-positive plasmid was constructed.

\section{Plasmid performance and absolute quantification}

We have generated a plasmid which contains a homozygous R882H mutation (Fig. 4). Significant differentiation to the wt DNA was possible up to a dilution of $10^{-1}$ copies. A copy number of $10^{-2}$ exceeded the threshold approximately at the cut-off value of 35 cycles (Fig. 5.a). However, the assay effectiveness was distinct when the plasmid containing a homozygous R882H mutation was compared with heterozygous patient samples. Two samples obtained from AML patients at diagnosis containing approximately $50 \%$ of DNMT3A R882H mutations exceeded the threshold at a similar cycle as the $10^{\circ}$ dilution of the plasmid (29.14/29.11 vs. 28.65; Fig. 5.b). The initial mutation portion was determined by Sanger sequencing. Since

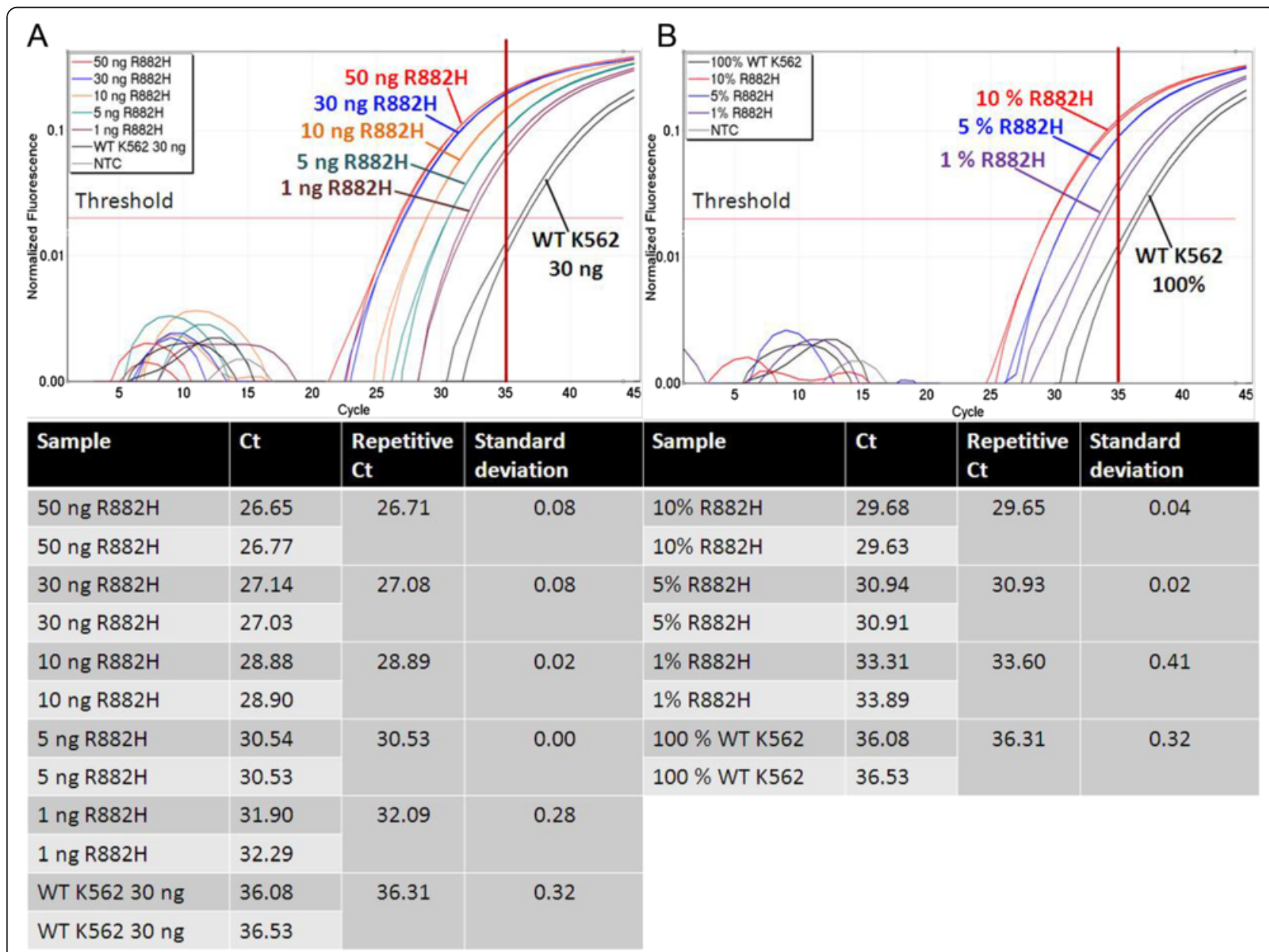

Fig. 3 Analysis of PCR sensitivity using serial dilutions of DNA (a) and (b) dilution of mutated DNA with wt DNA 
A

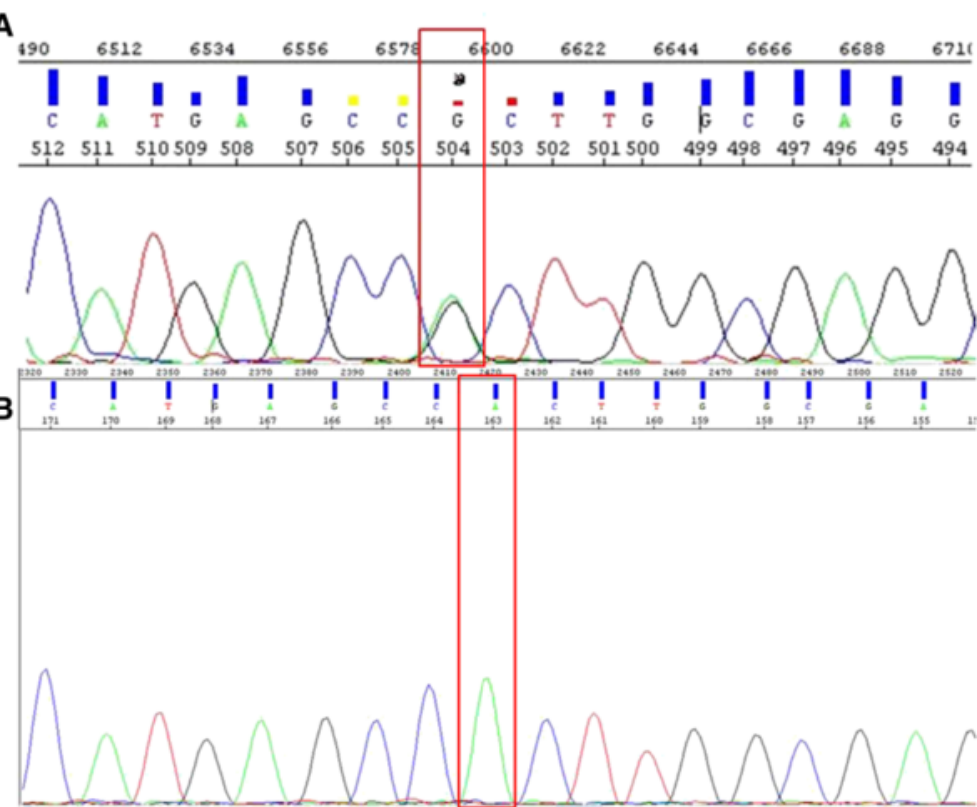

Fig. 4 Sequence of R882H plasmid. a Heterozygote R882H mutation in a patient sample. The mutational spot is indicated by the red box. Both, guanine (wt) and adenine (mutation) were detected. $\mathbf{b}$ Homozygote R882 $\mathrm{H}$ mutation in the generated plasmid. The mutational spot is indicated by the red box. The sequence displays only the mutated adenine base and no wt guanine base

the absolute quantification was not possible in this setting, we performed an absolute quantification by the percentage of mutated allele. Based on the previous measurement, the $10^{\circ}$ plasmid dilution was defined to contain $50 \%$ of DNMT3A R882H mutation. Fig. 5.c demonstrates a possibility of reliable quantification of patient samples using this setting. The sample containing $50 \%$ of DNMT3A R882H was detected at the same cycle as the $10^{\circ}$ plasmid dilution (25.15 vs. 25.07). In addition, the sample with $25 \%$ R882H mutation exceeded the threshold at a similar cycle compared to the $5^{*} 10^{-1}$ plasmid controls (26.33 vs. 26.1). The assay displayed a high efficiency between 0.98 and 1.03 (Fig. 5.b and d). The sensitivity of assay ranged up to $10^{-3}$.

In addition, follow-up samples of one patient were analyzed three times to examine the reproducibility of assay results. As shown in Table 2 the standard deviation was $\leq 3.1 \%$.

\section{Assays concordance}

Next, the concordance of different methods for detection of DNMT3A mutation was examined.

Initially, 25 samples from AML patients were analyzed using DNA sequencing. Next, endonuclease restriction analysis and ASB-PCR were used (Fig. 6). We have previously described that endonuclease restriction analysis has a perfect concordance with Sanger sequencing and is characterized by high sensitivity [22]. Qualitative assessment of band sizes (Fig. 6.a) corresponded to quantitative detection of $\mathrm{R} 882 \mathrm{H}$ mutation using ASB-
PCR (Fig. 6.b) in follow up samples from patient A, B, $\mathrm{C}$, und E. Patient D showed no DNMT3A R882H mutation using both methods. For example, increasing of DNMT3A R882H mutation in samples from patient $\mathrm{C}$ from $0.55 \%$ to $13.4 \%$ by ASB-PCR assay (Fig. 6.b) matched with a gain of band by endonuclease restriction analysis (Fig. 6.a). A cut-off of $\mathrm{Ct}=35$ was also applicable for analysis of follow-up samples. No false negative or positive signals were detected using the endonuclease restriction and ASB-PCR.

\section{Analysis of follow-up samples from AML patients}

We analyzed 62 follow up constitutive samples obtained from $6 \mathrm{AML}$ patients at diagnosis, after induction, consolidation therapy, and after alloSCT. All patients included in the follow-up analysis harbored a NPM1 mutation at diagnosis. This enabled comparison of DNMT3A stability during both $\mathrm{CR}$ and complete molecular remission (molCR) using the well-established marker for detection of MRD. The main characteristics, karyotype, concurrent mutations, percent of DNMT3A mutation, and MRD data of these patients are shown in Table 3. Median age was 55 years (range $43-60$ years), and the median survival time by the end of this study was 30 months (range 9-52 months).

For most analyzed patient, a DNMT3A R882H mutation was detectable after induction and consolidation therapy. The percentage of mutated allele was not constant and fluctuated in the course of disease. For example percentage of $\mathrm{R} 882 \mathrm{H}$ in follow-up samples of patient 6 ranged 


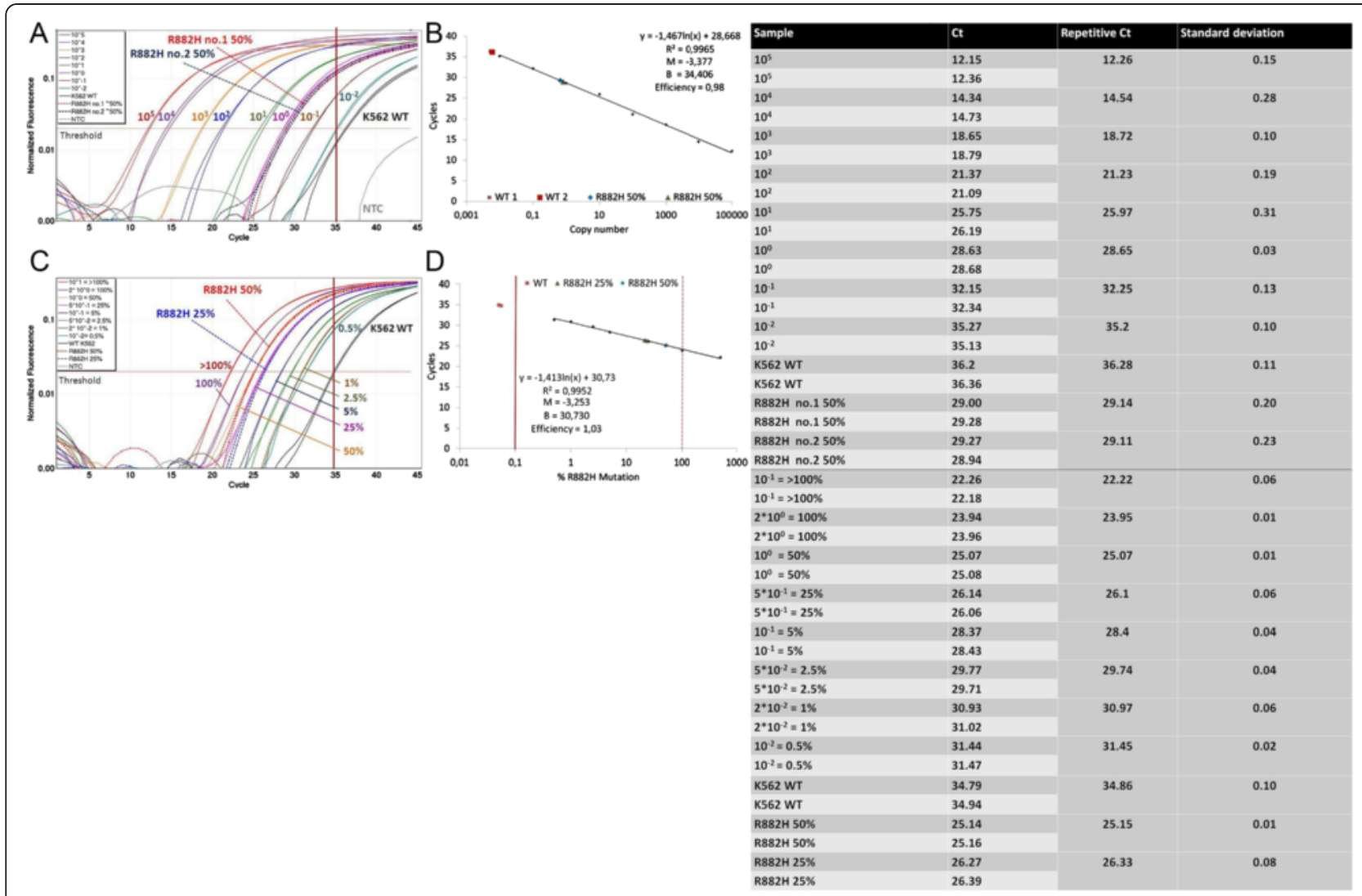

Fig. 5 Absolute quantification of DNMT3A R882H mutations. Values of ASB-PCR are listed in the corresponding table. a Analysis of copy number dilutions of the R882H homozygote plasmid. Patient samples with approximately $50 \%$ of R882H mutated allele are indicated by dotted lines; the $10^{\circ}$ plasmid dilutions are pink line. b The standard curve (copy numbers over cycle). Patient samples with $50 \%$ R882H are shown as a blue diamond and a green triangle. Wt samples are shown as red quadrats. $R^{2}=$ correlation coefficient. $B=$ Intercept with the ordinate. $M=S l o p e$ of the standard curve. c Absolute quantification was performed by percentage of mutated allele in a sample. Plasmid concentrations were adjusted to $50 \%$ and $25 \%$ of mutated R882H allele according to the measurement of heterozygote patient samples (dotted lines). $\mathbf{d}$ Standard curve (\%R882H over cycle). The dotted red line shows the limit of $100 \%$ R882H mutated allele in a sample. Patient samples are shown as a blue diamond and a green triangle. wt samples are shown as red quadrats. $R^{2}=$ correlation coefficienct. $B=$ Intercept with the ordinate. $M=$ Slope of the standard curve

from $0.05 \%$ to $4.05 \%$ during therapy. Furthermore, even in $\mathrm{CR}$ and molCR according to analysis of NPM1, a significant proportion of DNMT3A R882H mutation could be detected. In patients $1,2,3,4$, and 5 (Table 3), DNMT3A mutation was present at diagnosis and in CR after standard therapy. The amounts of mutation were decreased, for example, from $21.45 \%$ to $16.5 \%$ in patient 1 and from $14.95 \%$ to $1.2 \%$ in patient 4 . In patient 2 (Table 3), amount of mutated allele was later increased despite the patients was in CR with slight positive or negative MRD. The percentage of mutated allele was higher $(30.8 \%)$ as compared to diagnostic sample (11.95\%). At this time point, the patient exhibited thrombocytopenia in peripheral blood with normal blasts count in BM. In samples obtained in CR after alloSCT (Patients 1, 2, and 3, Table 3) with complete donor chimerism (CDC), DNMT3A mutation was not found. Interestingly, NPM1 mutation was found in very small amount. Overall, in CR no significant

Table 2 Reproducibility of ASB-PCR

\begin{tabular}{lllll}
\hline Sample & Run 1 (\%R882H) & Run 2 (\%R882H) & Run 3 (\%R882H) & Standard deviation (\%) \\
\hline 187 & 23.05 & 21.6 & 17.19 & 3.052 \\
$187-1$ & 8.2 & 8.65 & 8.4 & 0.225 \\
$187-2$ & 3.5 & 3.5 & 2.82 & 0.392 \\
$187-3$ & 2.1 & 1.4 & 1.07 & 0.525 \\
$187-4$ & 2.35 & 3.35 & 1.70 & 0.831 \\
$187-5$ & 31.35 & 33.05 & 35.3 & 1.981 \\
\hline
\end{tabular}




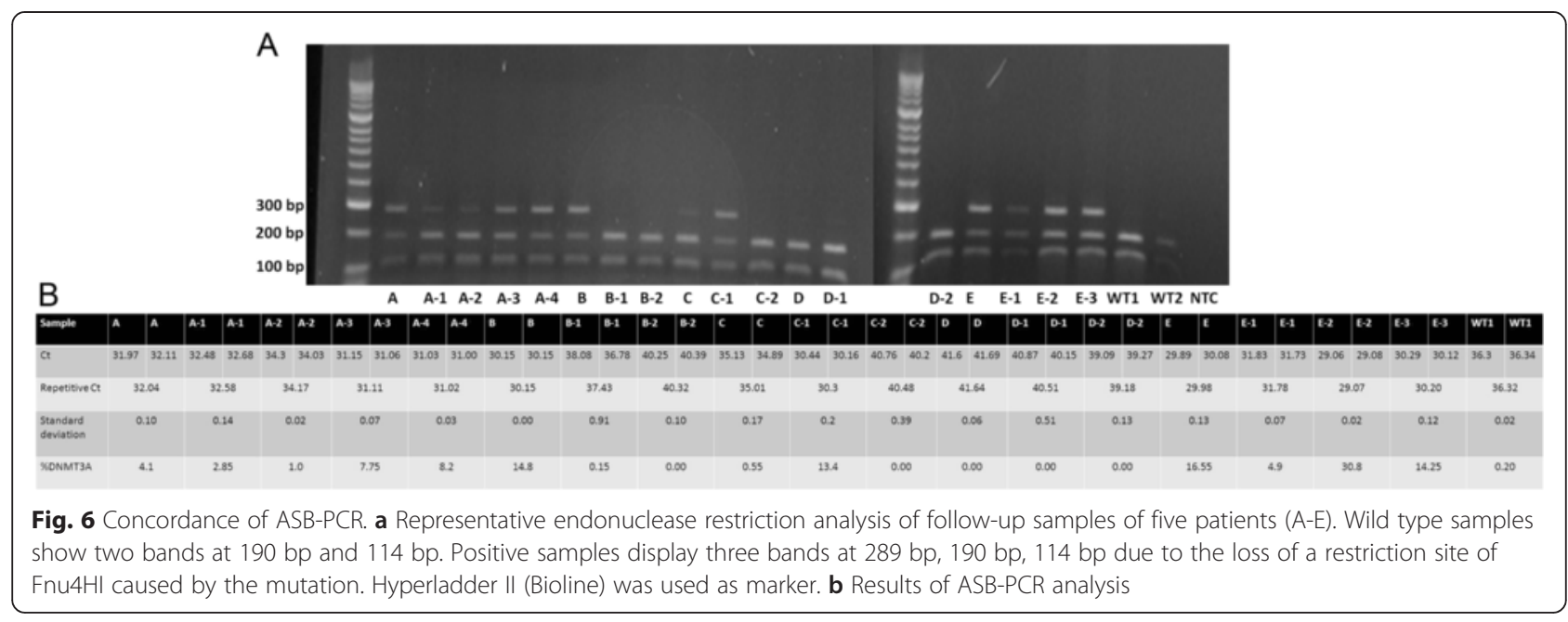

correlation between NPM1 and DNMT3A was detected. DNMT3A mutation was not detectable in patients 1,2 , and 3 after alloSCT, in CR with CDC. After alloSCT no DNMT3A R882H mutation was detected in cases when the patient displayed a complete or high percent of donor cells.

In relapse of AML, significant proportion of DNMT3A $\mathrm{R} 882 \mathrm{H}$ mutation was detected in all relapsed patients (Table 3). For instance, percentage of R882-positive allele increased significantly in patient 4 at relapse from $0 \%$ to $9.25 \%$ correlating with the molecular relapse detected by the percentage of NPM1 and reduced amount of donor cells $(10 \%)$. In patient 6 the decreasing of donor cells from $92 \%$ to $60 \%$ associated with a growing of R882-positive allele from $0 \%$ to $0.45 \%$. However, percentage of NPM1 did not increase and a relapse was not diagnosed. This patient underwent match related alloSCT after reduced intensity conditioning, and demonstrated delayed engraftment with slowly growing of donor cells. Notably, in the CR, the majority of patients showed a high level of DNMT3A mutations despite the low or negative rates of NPM1 mutation. In relapse, levels of both mutations have been increased.

\section{Discussion}

Last years, potential role of DNMT3A mutation for prognosis and outcome of AML has been extensively studied. Several authors have shown a negative impact of DNMT3A mutations on outcomes of AML patient [12, 13, 15, 18, 27-29]. DNMT3A mutation has been shown an independent poor prognostic factor for overall survival and relapse-free survival [15]. Currently, only few methods for the quantitative detection of DNMT3A R882H mutations are available $[19,30]$. Here, we developed an ASB-PCR assay that allows discrimination between wt and mutated DNMT3A and quantification of $\mathrm{R} 882 \mathrm{H}$ allele in one reaction. The main feature of this assay is the combination of an allele-specific primer with a competitive blocker as described by Morlan et al. [25]. Features of DNMT3A R882H sequence allowed introduction of a purin-purin-mismatch (G-A) to the allele-specific primer leading to its high selectivity. Thus, a significant amplification difference for the wt and mutant allele was seen also without the use of the competitive blocker. Adding blocker enhanced selective amplification of R882Hmutated allele that lead to earlier detection of mutation. Furthermore, the maximum $C_{t}$ difference between wt and mutated allele was 14 cycles. Thereby, effectiveness of the blocker is caused by intersection with the allele-specific primer and location of the discriminating base in the middle of the blocker sequence. In contrast, the allele-specific primer includes the discriminating base at its 3 '-end.

In addition to the enabling of discrimination between wt and mutant allele, the assay had to facilitate analysis of follow-up samples that contain a low percentage of mutated allele. The high sensitivity of $10^{-3}$ and the possibility to use low DNA amounts, up to $1 \mathrm{ng}$, make this approach feasible.

Here, absolute quantification was performed by the percentage of mutated $\mathrm{R} 882 \mathrm{H}$ allele in a sample. Since the generated plasmid contained a homozygous $\mathrm{R} 882 \mathrm{H}$ mutation the amplification characteristics using ASBPCR were different as compared to patient samples containing the heterozygous mutation. Possibly, the missing competition between wt and mutant allele during the amplification of plasmid DNA leads to a more efficient enrichment of mutant R882H generating higher $\mathrm{C}_{t}$ values. Modification of plasmid concentrations according to the percentage of mutated allele in the patient samples determined by sequencing enabled a reliable absolute quantification. Alternatively, the generation of an additional plasmid containing the wt allele is possible. Absolute quantification can be performed by combining the wt plasmid with different proportions of the plasmid containing the mutated allele [31]. 
Table 3 Quantitative analysis of DNMT3A R882H in follow-up samples from 6 AML patients

\begin{tabular}{|c|c|c|c|c|c|c|c|c|c|}
\hline Patient & Age/Gender & $F A B$ & Karyotype & $\begin{array}{l}\text { Mutations at } \\
\text { diagnosis }\end{array}$ & Disease status & DNMT3A \% & NPM1 log & FLT3 Ratio & Outcome/OS \\
\hline \multirow[t]{4}{*}{1} & $52 / F$ & M4 & $46, X X$ & NPM1 & DS & 21,45 & $1 \wedge 10^{6}$ & 0,72 & $\mathrm{CR} / 9+$ \\
\hline & & & & FLT3-ITD & $\mathrm{CR}$ after $\mathrm{CT}$ & 16,4 & $1 \wedge^{-5}$ & 0 & \\
\hline & & & & FLT3-TKD2 & CR, day+28 after alloSCT, CDC & 0 & 0 & 0 & \\
\hline & & & & DNMT3A & CR, day+195 after alloSCT, CDC & 0 & 0 & 0 & \\
\hline \multirow[t]{9}{*}{2} & $60 / M$ & M2 & $46, X Y$, del $(9 q)$ & NPM1 & DS & 11,95 & $1 \wedge 10^{5}$ & & $C R / 31+$ \\
\hline & & & & DNMT3A & CR after IT & 5,8 & $1 \wedge 10^{-2}$ & & \\
\hline & & & & & $\mathrm{CR}$ after $\mathrm{CT}$ & 0,05 & $1 \wedge 10^{-4}$ & & \\
\hline & & & & & CR, Platelet $\downarrow$ & 0,1 & $1 \wedge 10^{-4}$ & & \\
\hline & & & & & $C R$ & 16,5 & $1 \wedge 10^{-5}$ & & \\
\hline & & & & & $C R$ & 30,8 & 0 & & \\
\hline & & & & & $C R$ & 11,3 & $1 \wedge 10^{-4}$ & & \\
\hline & & & & & CR, day+26 after alloSCT, $98 \%$ DC & 0 & 0 & & \\
\hline & & & & & CR, day+73 after alloSCT (CDC) & 0 & 0 & & \\
\hline \multirow[t]{5}{*}{3} & $43 / F$ & M4 & $46, X X$ & NPM1 & DS & 17,9 & $1 \wedge 10^{5}$ & 0,78 & $C R / 52+$ \\
\hline & & & & FLT3-ITD & $\mathrm{CR}$, day+563 after alloSCT, CDC & 0 & $1 \wedge 10^{-5}$ & 0 & \\
\hline & & & & DNMT3A & $\mathrm{CR}$, day+731 after alloSCT, CDC & 0 & $1 \wedge 10^{-4}$ & 0 & \\
\hline & & & & & CR, day+1082 after alloSCT, CDC & 0 & 0 & 0 & \\
\hline & & & & & CR, day+1445 after alloSCT, CDC & 0 & 0 & 0 & \\
\hline \multirow[t]{8}{*}{4} & $50 / \mathrm{M}$ & M4 & $46, X Y$ & NPM1 & DS & 14,95 & $1,0 \wedge 10^{5}$ & 0,41 & $\mathrm{R} / 17+$ \\
\hline & & & & FLT3-ITD & CR after IT & 1,6 & $1 \wedge 10^{-4}$ & 0 & \\
\hline & & & & DNMT3A & $C R$ after $C T$ & 1,2 & $1 \wedge 10^{-5}$ & 0 & \\
\hline & & & & & CR, day+40 after alloSCT, CDC) & 0 & $1 \wedge 10^{-4}$ & 0 & \\
\hline & & & & & CR, day+130 after alloSCT, CDC & 0,1 & 0 & 0 & \\
\hline & & & & & $C R$, day+217 after alloSCT, CDC & 0 & 0 & 0 & \\
\hline & & & & & $\begin{array}{l}\text { R, day+306 after alloSCT, } \\
10 \% \text { DC }\end{array}$ & 9,25 & $1^{\wedge} 10^{5}$ & 0 & \\
\hline & & & & & R, day+373 after alloSCT, $15 \%$ DC & 11,7 & $1 \wedge 10^{4}$ & 0,25 & \\
\hline \multirow[t]{10}{*}{5} & $50 / F$ & $5 a$ & $46, X X$ & NPM1 & DS & 22,15 & $1 \wedge 10^{5}$ & 0,5 & $C R / 26$ \\
\hline & & & & FLT3-ITD & CR after IT & 0,35 & $1 \wedge 10^{-4}$ & 0 & \\
\hline & & & & DNMT3A & CR after IT & 0,2 & $1 \wedge 10^{-4}$ & 0 & \\
\hline & & & & IDH1 & $C R$ after $C T$ & 0,1 & $1 \wedge 10^{-4}$ & 0 & \\
\hline & & & & & $\mathrm{CR}$ after $\mathrm{CT}$ & 0,05 & 0 & 0 & \\
\hline & & & & & CR, day+29 after alloSCT, CDC & 0,05 & $1 \wedge 10^{-4}$ & 0 & \\
\hline & & & & & CR, day+71 after alloSCT, CDC & 0 & $1 \wedge 10^{-5}$ & 0 & \\
\hline & & & & & $\begin{array}{l}\text { R, day+114 after alloSCT, } \\
90 \% \mathrm{DC}\end{array}$ & 0,05 & $1 \wedge 10^{-4}$ & 0 & \\
\hline & & & & & CR, day+174 after alloSCT, CDC & 0 & 0 & 0 & \\
\hline & & & & & $C R$, day+215 after alloSCT, CDC & 0 & 0 & 0 & \\
\hline \multirow[t]{5}{*}{6} & $71 / \mathrm{M}$ & $5 a$ & $46, X Y$ & NPM1 & DS & 12,75 & $1 \wedge 10^{4}$ & & $C R / 46+$ \\
\hline & & & & DNMT3A & CR after IT & 0,1 & $1 \wedge 10^{-2}$ & & \\
\hline & & & & $\mathrm{IDH} 1$ & $\mathrm{CR}$ after $\mathrm{CT}$ & 0,1 & $1 \wedge 10^{-4}$ & & \\
\hline & & & & IDH2 & $\mathrm{CR}$ after $\mathrm{CT}$ & 1,65 & $1 \wedge 10^{-4}$ & & \\
\hline & & & & & $C R$ & 0,1 & $1 \wedge 10^{-4}$ & & \\
\hline
\end{tabular}


Table 3 Quantitative analysis of DNMT3A R882H in follow-up samples from 6 AML patients (Continued)

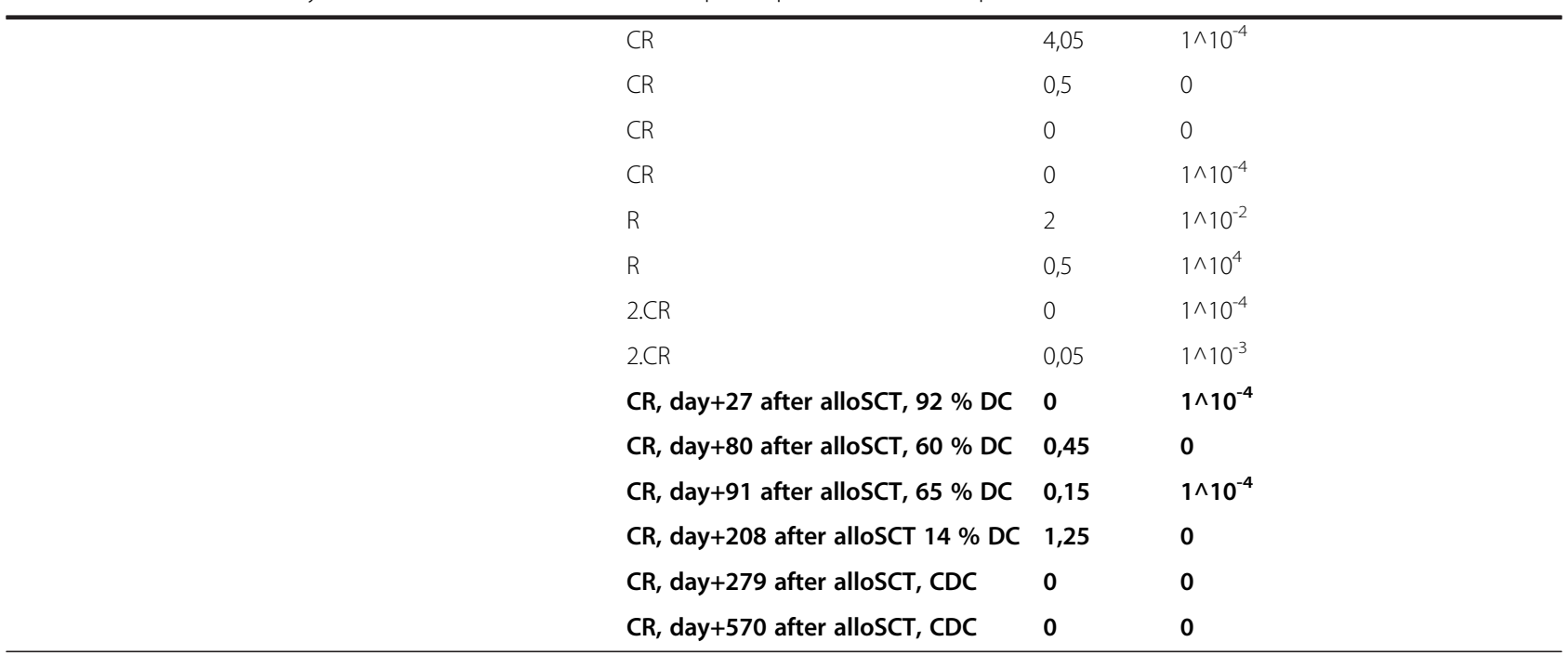

$F A B$ French-American-British classification systems, DS primary diagnosis, IT induction therapy, $C T$ consolidation therapy, $C R$ complete remission, $R$ Relapse, alloSCT allogeneic stem cell transplantation, $C D C$ complete donor chimerism, $D C$ donor cells

+patient alive at the end of study. Samples after alloSCT are bold

For application of ASB-PCR in diagnostic routine the concordance with other analysis methods is important. ASB-PCR displayed a perfect concordance with sequencing and endonuclease restriction analysis [22]. This accounts for a high specificity of the assay. However, because low amplification of wt samples is present a wt control should be run together with patient samples. Here, the cut-off value of $C_{t}=35$ was applicable in every run but this could depend on the PCR reagents and machine used for Real-Time quantification.

To demonstrate the need and effectiveness of new established method, we analyzed 62 follow-up samples from 6 AML patients after therapy and alloSCT. Moreover, data about the stability of DNMT3A mutation during the course of disease are restricted and controversial. Previously published studies have demonstrated that DNMT3A mutations are stable at relapse $[12,15]$. Thol et al. found that the mutations disappeared at CR and recurred at relapse in one patient [12]. Later, Hou et al. reported persistence of DNMT3A mutations at CR in 5 patients, which later achieved relapse and died of disease progression. These data could relate the persistence of DNMT3A mutations and high risk of relapse [18]. Recently, Pløen et al. have identified persistence of DNMT3A mutations in long-term remission of patients with AML that received cytoreduction or palliative therapy [19]. Using cell-sorting, the authors showed that DNMT3A mutations were present in $\mathrm{T}$-cells and $\mathrm{B}$-cells at diagnosis in some patients, and also in T-cells several years after diagnosis. The presence of DNMT3A in both B-cells and T-cells could lead to the assumption that mutation had occurred in an early pre-leukemic stem cell prior to the acquisition of other genetic events, and could be resistant to chemotherapy [19]. Therefore, further exploration of the role of DNMT3A R882H mutations for the progression of AML disease is needed.

In accordance with previous studies, we found persistence of DNMT3A R882H mutations after standard cytoreduction therapy. In most cases the amount of mutated allele was lower as compared with the diagnostic sample. But in one case (Patient 2, Table 3) we have found an increasing of DNMT3A R882H allele in CR without relapse signs. Furthermore, NPM1 remained negative indicating potential persistence of a different clone. The permanent presence of DNMT3A R882H after therapy in deep CR could be indicating presence of DNMT3A mutation in early pre-leukemic stem cells that resist chemotherapy. This could also explain the loss of correlation between NPM1 and DNMT3A because the clonal expansion of pre-leukemic stem cells could originate independent of NPM1 positive clones [10, 11]. In relapse, all samples showed an increasing of both NPM1 and DNMT3A mutated alleles. This suggests at least in part the presence of NPM1 and DNMT3A mutations in the same cell clone. After alloSCT in patients in CR with complete donor chimerism we have no found DNMT3A $\mathrm{R} 882 \mathrm{H}$. This data suggests the removal of leukemic stem cells after alloSCT and indicates the importance of alloSCT for high risk AML patients.

In summary, we developed a rapid, sensitive and specific method for quantitative detection of DNMT3A R882H mutations in AML patients. This assay could be easily applicable for routine screening of DNMT3A R882 mutation not only at time of diagnosis but also after the treatment. Quantitative detection of DNMT3A R882H mutations at different time points of AML disease enables screening of 
follow-up samples. This could assist to evaluate response to therapy and provide additional information about the role of DNMT3A mutations in development and progression of AML.

\section{Competing interests}

The authors declare that they have no competing interests.

\section{Authors' contributions}

RB designed and carried out the molecular genetic studies, drafted the manuscript. IWB designed research and revised the manuscript. NS carried out the molecular genetic studies. CB, AP, and BD participated in the design of study and revised the manuscript. OB designed research, carried out the sequencing analyses and revised the manuscript. All authors read and approved the final manuscript.

\section{Acknowledgment}

This work was supported by the Stefan-Morsch-Stiftung for Leukemia Tumour Patients.

Received: 25 February 2015 Accepted: 12 May 2015

Published online: 22 May 2015

\section{References}

1. Marcucci G, Metzeler KH, Schwind S, Becker H, Maharry K, Mrozek K, et al. Age-related prognostic impact of different types of DNMT3A mutations in adults with primary cytogenetically normal acute myeloid leukemia. I Clin Oncol. 2012;30(7):742-50.

2. Ley TJ, Ding L, Walter MJ, McLellan MD, Lamprecht T, Larson DE, et al. DNMT3A mutations in acute myeloid leukemia. N Engl J Med. 2010;363(25):2424-33.

3. Zhang Y, Chen FQ, Sun YH, Zhou SY, Li TY, Chen R. Effects of DNMT1 silencing on malignant phenotype and methylated gene expression in cervical cancer cells. J Exp Clin Cancer Res. 2011;30:98.

4. Jasielec J, Saloura V, Godley LA. The mechanistic role of DNA methylation in myeloid leukemogenesis. Leukemia. 2014;28(9):1765-73.

5. Li KK, Luo LF, Shen Y, Xu J, Chen Z, Chen SJ. DNA methyltransferases in hematologic malignancies. Semin Hematol. 2013;50(1):48-60.

6. O'Brien EC, Brewin J, Chevassut T. DNMT3A: the DioNysian MonsTer of acute myeloid leukaemia. Ther Adv Hematol. 2014;5(6):187-96.

7. Holz-Schietinger C, Matje DM, Reich NO. Mutations in DNA methyltransferase (DNMT3A) observed in acute myeloid leukemia patients disrupt processive methylation. J Biol Chem. 2012;287(37):30941-51.

8. Russler-Germain DA, Spencer DH, Young MA, Lamprecht TL, Miller CA, Fulton $\mathrm{R}$, et al. The R882H DNMT3A mutation associated with AML dominantly inhibits wild-type DNMT3A by blocking its ability to form active tetramers. Cancer Cell. 2014;25(4):442-54.

9. McDevitt MA. Clinical applications of epigenetic markers and epigenetic profiling in myeloid malignancies. Semin Oncol. 2012;39(1):109-22.

10. Shlush LI, Zandi S, Mitchell A, Chen WC, Brandwein JM, Gupta V, et al. Identification of pre-leukaemic haematopoietic stem cells in acute leukaemia. Nature. 2014;506(7488):328-33.

11. Corces-Zimmerman MR, Hong WJ, Weissman IL, Medeiros BC, Majeti R. Preleukemic mutations in human acute myeloid leukemia affect epigenetic regulators and persist in remission. Proc Natl Acad Sci U S A. 2014;111(7):2548-53.

12. Thol F, Damm F, Ludeking A, Winschel C, Wagner K, Morgan M, et al. Incidence and prognostic influence of DNMT3A mutations in acute myeloid leukemia. J Clin Oncol. 2011;29(21):2889-96.

13. Ribeiro AF, Pratcorona M, Erpelinck-Verschueren C, Rockova V, Sanders M, Abbas $\mathrm{S}$, et al. Mutant DNMT3A: a marker of poor prognosis in acute myeloid leukemia. Blood. 2012;119(24):5824-31.

14. Ibrahem L, Mahfouz R, Elhelw L, Abdsalam EM, Soliman R. Prognostic significance of DNMT3A mutations in patients with acute myeloid leukemia. Blood Cells Mol Dis. 2015;54(1):84-9.

15. Im AP, Sehgal AR, Carroll MP, Smith BD, Tefferi A, Johnson DE, et al. DNMT3A and IDH mutations in acute myeloid leukemia and other myeloid malignancies: associations with prognosis and potential treatment strategies. Leukemia. 2014;28(9):1774-83.
16. Shivarov V, Gueorguieva R, Stoimenov A, Tiu R. DNMT3A mutation is a poor prognosis biomarker in AML: results of a meta-analysis of $4500 \mathrm{AML}$ patients. Leuk Res. 2013;37(11):1445-50.

17. Wakita S, Yamaguchi H, Omori I, Terada K, Ueda T, Manabe E, et al. Mutations of the epigenetics-modifying gene (DNMT3a, TET2, IDH1/2) at diagnosis may induce FLT3-ITD at relapse in de novo acute myeloid leukemia. Leukemia. 2013;27(5):1044-52.

18. Hou HA, Kuo YY, Liu CY, Chou WC, Lee MC, Chen CY, et al. DNMT3A mutations in acute myeloid leukemia: stability during disease evolution and clinical implications. Blood. 2012;119(2):559-68.

19. Ploen GG, Nederby L, Guldberg P, Hansen M, Ebbesen LH, Jensen UB, et al. Persistence of DNMT3A mutations at long-term remission in adult patients with AML. Br J Haematol. 2014;167(4):478-86.

20. Genovese G, Kahler AK, Handsaker RE, Lindberg J, Rose SA, Bakhoum SF, et al. Clonal hematopoiesis and blood-cancer risk inferred from blood DNA sequence. N Engl J Med. 2014;371(26):2477-87.

21. Jaiswal S, Fontanillas P, Flannick J, Manning A, Grauman PV, Mar BG, et al. Age-related clonal hematopoiesis associated with adverse outcomes. N Engl J Med. 2014;371(26):2488-98.

22. Berenstein R, Blau IW, Kar A, Cay R, Sindram A, Seide C, et al. Comparative examination of various PCR-based methods for DNMT3A and IDH1/2 mutations identification in acute myeloid leukemia. J Exp Clin Cancer Res. 2014;33:44.

23. Vardiman JW, Thiele J, Arber DA, Brunning RD, Borowitz MJ, Porwit A, et al. The 2008 revision of the World Health Organization (WHO) classification of myeloid neoplasms and acute leukemia: rationale and important changes. Blood. 2009;114(5):937-51.

24. Jaatinen T, Laine J. Isolation of mononuclear cells from human cord blood by Ficoll-Paque density gradient. Curr Protoc Stem Cell Biol. 2007;Chapter 2:Unit 2A.1. doi:10.1002/9780470151808.sc02a01s1.

25. Morlan J, Baker J, Sinicropi D. Mutation detection by real-time PCR: a simple, robust and highly selective method. PLoS ONE. 2009;4(2), e4584.

26. Blau O, Berenstein R, Sindram A, Blau IW. Molecular analysis of different FLT3ITD mutations in acute myeloid leukemia. Leuk Lymphoma. 2013;54(1):145-52.

27. Lu Q, Chen Y, Wang H, Li Z. DNMT3A mutations and clinical features in Chinese patients with acute myeloid leukemia. Cancer Cell Int. 2013;13(1):1.

28. Markova J, Michkova P, Burckova K, Brezinova J, Michalova K, Dohnalova A, et al. Prognostic impact of DNMT3A mutations in patients with intermediate cytogenetic risk profile acute myeloid leukemia. Eur J Haematol. 2012;88(2):128-35.

29. Schwarz J, Markova J. DNMT3A mutations in AML: a new prognostic factor? Leuk Res. 2013;37(11):1432-3.

30. Shivarov V, Ivanova M, Naumova E. Rapid detection of DNMT3A R882 mutations in hematologic malignancies using a novel bead-based suspension assay with BNA(NC) probes. PLoS ONE. 2014;9(6), e99769.

31. Szankasi P, Reading NS, Vaughn CP, Prchal JT, Bahler DW, Kelley TW. A quantitative allele-specific PCR test for the BRAF V600E mutation using a single heterozygous control plasmid for quantitation: a model for qPCR testing without standard curves. J Mol Diagn. 2013;15(2):248-54.

\section{Submit your next manuscript to BioMed Central and take full advantage of:}

- Convenient online submission

- Thorough peer review

- No space constraints or color figure charges

- Immediate publication on acceptance

- Inclusion in PubMed, CAS, Scopus and Google Scholar

- Research which is freely available for redistribution 